\title{
8 Conclusion
}

\section{Towards a research agenda for knowledge co-production in Urban Africa}

\author{
Sylvia Croese
}

This book brings together contributions from researchers and practitioners across Africa to provide insight into some of the continent's most pressing urban development challenges, including infrastructural inequality, affordable housing, climate resilience, and food security. The chapters show how African cities - ranging from small, peri-urban areas to entire mega-city regions - are at the coalface of such challenges. Central to all the cases is the disjuncture between policy and practice, often resulting from contrasting needs, understandings, and interests that guide the actions of both states and citizens. This disjuncture produces complex spaces and arrangements in which formal plans and policies are intertwined with informal actors and practices that are in turn strongly shaped by local histories and politics.

Taken together, the complex reality of contemporary governance in African cities has a daily impact on the degree to which cities and communities are safe, inclusive, resilient, and sustainable, and able to meet local and global urban development goals. The need for knowledge that engages meaningfully with this complexity and is capable of capturing the finer and often shifting grain of informal systems and actors is urgently needed to inform improved policies, governance, and urban development planning. By bringing together multiple actors and perspectives to examine and address these challenges and their different manifestations in eight African cities, the collection's authors created new spaces and entry points for knowledge sharing, production, and experimentation. This concluding chapter reflects and builds on those contributions, with the aim of outlining a research agenda to guide ongoing and future knowledge co-production in urban Africa.

\section{Practising knowledge co-production in urban Africa}

There is no single definition of knowledge co-production, and much of the literature on co-production still focuses on the co-production of services (Mitlin \& Bartlett, 2018). Moreover, while there is increasing interest and work on transdisciplinary knowledge co-production, particularly in the field of (urban) sustainability, this still remains a novel approach to research in Africa, with the exception of 
South Africa (Swilling, 2014). ${ }^{1}$ As such, this collection's main purpose and contribution is to share its experimental practice of alternative and innovative forms of knowledge generation through co-production in urban Africa.

Most of the chapters in this volume follow a CityLab approach, broadly understood as the creation of deliberative spaces aimed at enabling dialogue between different urban actors and stakeholders. As such, many of the researchers conducted multi-stakeholder roundtables, meetings and workshops, municipal fora, learning labs, and policy dialogues. Other research methods included secondary literature review, participatory mapping, personal interviews, focus group discussions, GIS-enabled surveys, direct observations, as well as quantitative datagathering methods.

To ensure the inclusivity and relevance of the research, all of these tools and methods included a vast range of stakeholders, from city and other (local) government officials, traditional leadership (chiefs), community leaders, and political party representatives, to local community organization representatives, business owners, and ordinary citizens, including youth. As a process-driven enterprise, the undertaking of urban knowledge co-production required not only the cooperation of stakeholders, but also their inclusion as equal research partners with invaluable perspectives. In most cases, the research also necessarily included activities ranging from community sensitization, stakeholder mapping and identification, and other preparatory meetings in the inception phase, to end-of-project stakeholder meetings and presentations as part of the research completion phase.

In addition to the chapters that focus on knowledge co-production within individual cities, a number of chapters focus on co-producing knowledge across geographical boundaries by comparing different cities within the same country (Chapter 3) or across national borders (Chapter 5). These comparative studies intend to offer yet another way of generating questions and insights to contribute to the construction of new 'South-South' knowledges, outside of the traditional paradigms of research practice and thought. While both singular (i.e., looking at one case city) and comparative modes of knowledge co-production offer productive avenues for rich insights into local realities, they are not without their challenges. The chapters here show that when working within a single local community, challenges related to (the lack of) trust between and among the varied urban stakeholders and complex local governance structures operating in that space can pose significant barriers. This dynamic was particularly notable in instances where power is practised and shared by numerous actors both in and outside of formal state structures (e.g., political parties and traditional authorities). Meanwhile, when engaged in comparative urbanism research, other practical challenges emerged, such as dealing with differences in definitions (e.g., of informality), expectations, and data sources and reliability from city to city.

Taken together, the chapters in this volume show the need for much more conscious reflection on the specific challenges involved in co-producing knowledge in urban Africa in order to come up with definitions and methods that are suitable and appropriate to Africa's context-specific local realities. In doing so, there is 
much to be built on in terms of the different exercises of adjustment and experimentation that the researchers in this book demonstrate to have undertaken in responding to and overcoming their respective sets of local challenges.

As the authors of Chapter 2 argue when writing about Lusaka's Soweto Market, the complexity of local governance structures has tangible consequences for the extent to which stakeholders can freely participate in (spaces of) governance, including spaces of knowledge co-production. Researchers thus must have the knowledge, intuition, and skills required to navigate and manage such spaces. As such, the ability to assess the degree of 'freedom' needed for participants to express themselves, and/or create conditions to facilitate the co-production process are crucial, and will mean different things in different contexts, as is also demonstrated in Chapters 4 and 6 (Thika and Kumasi). ${ }^{2}$

With these concerns in mind, the authors of Chapter 2 describe how they chose to 'separate political leaders from the administrative staff of the Lusaka City Council during the two CityLabs held on Soweto Food Market, in order for them to engage more freely' (my italics). Similarly, the authors of Chapter 4 on land sharing in Thika describe how workshop participants were chosen 'by gender and age, creating one workshop for women, one for youth, and one for men. The aim [being] to enable optimal engagement in a free atmosphere, and to better capture divergent and sometimes gender-specific tenure issues' (my italics). Workshop participants in Sepe-Buokrom in Kumasi, Ghana were similarly divided into groups based on age, gender, and power of authority in order for participants to express themselves freely (my italics). Despite these efforts, certain hierarchies persisted even within the groupings (e.g., 'queen mothers' within the women's focus group in Kumasi, and representatives of the chief in the men's group). That said, researchers continued to do what they could to ensure participants' comfort and ability to express themselves, as was seen in the Kumasi CityLab, where the local language (Twi) was used as the medium of communication during all focus group discussions at the community level for maximum participant involvement and understanding (Chapter 6).

The meaning and impact of such adjustments and interventions cannot be taken for granted, and depend on researchers being both in tune with local realities and flexible enough to adjust and switch gears when needed. There is a need for further analysis of the 'practice of best practice' as used throughout the continent, and the kind of tactical knowledge that underpins such work (Bulkeley, 2006). Such analysis would provide an important basis for further experimentation, learning, and theorization of alternatives to universal 'best practices' of knowledge co-production based on local experiences in urban Africa (Patel et al., 2015), as well as for a research agenda that can support and strengthen such endeavours.

\section{Towards a research agenda}

Existing practices of knowledge co-production in urban Africa show the importance of experimentation and innovation with alternative forms of knowledge 
generation. These are not limited to crossing societal, disciplinary, linguistic, sectoral, or geographical boundaries, but also include the use and mixing of new technologies to support 'citizen science' (West et al., 2020).

How can the experience gained, the knowledge applied, and the social capital built in co-production experiments by both researchers and co-production partners be harnessed? How can an explicit link to policy formulation and implementation be made? And how can sustained modes of collaboration be forged in order to build a lasting bridge between research and policy? These are some of the questions that we hope this collection catalyses researchers and urban practitioners to explore further.

\section{Supporting the role and capacity building of researchers}

Most of the contributors to this book have a vast wealth of knowledge and research experience, which we argue is a vital asset in navigating the complexity of knowledge co-production processes. Many of the authors refer to the importance of continuous engagement with stakeholders, as well as the neutral positionality of the researchers as partners, in order to build and maintain trust. The consequence of these deliberate efforts is the creation of an atmosphere conducive to participatory and inclusive dialogue (Chapters 4 on Thika and 7 on Luanda). In addition, the authors stress the ability to identify key actors with whom future partnerships could be developed (e.g., Chapter 6 on Kumasi) - a type of tacit relational knowledge that is acquired through years of experience and practice rather than the application of scientific or academic protocols.

How can such roles be better supported, and how can the knowledge created in these processes be captured and passed on to new generations of researchers? Importantly, the Centre for Urban Research and Planning at the University of Zambia (Chapter 2) and the Centre for Urban Research and Innovations of the University of Nairobi (Chapter 4) are members of the Association of Africa Planning Schools (AAPS), which intends to transform urban planning education and practice in Africa by equipping up-and-coming urban planners with the relevant skills and methodologies to address the challenges facing the African city. As demonstrated across the different chapters in this volume, this is crucial work not only for research to more effectively contribute to participatory and inclusive community plans and policies, but also to strengthen the research-education nexus (Duminy et al., 2014).

\section{Engaging knowledge co-production partners}

The chapters in this book have illustrated the importance of relationship building and management in the knowledge co-production process. This not only was an essential research tool, but also in itself an important process that provided the researchers with deeper insight and understanding of the perceptions and experiences of research stakeholders. 


\section{Sylvia Croese}

The researchers in Kumasi, Ghana point to important nuances that were uncovered through the co-production process used in focus group discussions and stakeholder meetings, such as the community's diversified nature based on ethnic lines, apparent rivalry and tensions between local leaders, and various perspectives on the potential for and challenges to successful implementation of a Community Resilience Framework (CRF) for flood-risk management (Chapter 6). Consciousness of these nuances and the ability to engage with them carefully and constructively allowed the researchers to contribute to building awareness within the community of its own ability to take initiative independently of government action. That said, despite efforts to ensure a gender balance in stakeholder groups, the group composition in this research was overwhelmingly male, with females constituting just over one third of the participants. According to the authors, this is reflective of the male-dominated nature of Ghanaian culture, particularly in areas of decision-making. This observation raises questions around how such perceptions can be overcome, and how co-production processes can be more inclusive of gender and age, as well as political and religious beliefs.

Working with numerous partners builds greater and more nuanced understanding of the challenges faced by the different stakeholders. This is seen in the case of Zambian public servants who deal with the daily politics of urban markets, but also must strive to remain professional (Chapter 2) - an example that speaks to the plight of the countless public servants caught between the pressures emanating from failing market economy models playing out in constitutional democracies. The resulting conflicts and paradoxes of that dynamic are also noted in Chapter 5's comparative study of Johannesburg and Cairo, where 'on one hand, [there is] the need for cost recovery, and on the other, a constitutional pledge guaranteeing citizens the right to these services - [a situation that] has led to a number of court cases'.

In some cases, the political weight associated with key challenges can mean that government officials feel they need to withdraw themselves from the work, as experienced by the authors of Chapter 4 working on the 'the land question' in Thika, Kenya - a question that was considered 'too emotive to engage in during a campaign period'. In the same project, which examined the potential for land sharing in an informal settlement, the researchers also had to accommodate the high turnover of government officials, and the effects of shifts in political leadership on potential gains made under previous administrations. The tension between the long-term process of knowledge co-production and short-term political cycles represents an often unavoidable barrier to extending co-production models to regional and national levels, and putting results to action.

Such barriers also point to the necessity of taking things like political election cycles into account when planning research, and devising methodologies to engage high-level government officials and to mitigate against political influence. The case of Luanda (Chapter 7) is instructive in this regard. Working with both community members and local government officials through training and capacity building for data collection, this co-produced effort contributed not only to 
stakeholders having a better understanding of the intentions behind the work, but also to a local sense of ownership of the data and knowledge produced. Also seeking to fully engage focus group discussion participants in Kumasi, Ghana (Chapter 6), researchers note the positive impact of beginning all sessions with an audio-visual presentation of flood events, which moved participants to actively contribute to the discussions that followed. Whichever tools or methods were employed, all of the chapters in this volume repeatedly illustrated the vital importance of seeing co-production through to the end: that is, communicating and sharing research results as part of community and stakeholder engagement.

\section{The research-policy nexus}

One of the key questions underlying and unifying this volume is how coproduced research can be harnessed to inform both local and global development policies and agendas. Here we must take care to not blindly mimic global agendas - that is, simply transposing them to the local level - but rather to critically evaluate the relevance and utility of these agendas, and accordingly change and adapt them where necessary. To that end, as was revealed by some of the research here, co-production represents a useful tool to localize global development frameworks such as the Sustainable Development Goals (SDGs) and New Urban Agenda (NUA). Notably, several chapters revealed important gaps and shortcomings of global and local development agendas, by highlighting the importance of good land governance and well-functioning land administration systems (Chapter 4), spatial and intra-city inequality (Chapter 5), and the importance of assessing the extent to which existing urban policies do not leave anyone behind (Chapter 7).

In critically interrogating development agendas at the finer grain of local-level real-world application and through the lens of a multitude of stakeholders, all chapters underscore the importance of the negotiated co-production of policy interventions, as opposed to policies that are either imposed from top to bottom (or vice versa) without taking into account the full spectrum of urban actors, as well as practices and systems of urban informality, that operate in African cities on a daily basis. As such, the authors of Chapter 3 argue for the adoption of an integrative policy approach that necessarily considers and builds upon the existing ways in which the formal and informal are both connected and disconnected across three cities in Egypt. Similarly, authors of Chapter 6 stress the importance of Community Resilience Frameworks (CRFs) for understanding how communities at high risk of flooding effectively perceive their own adaptive preparedness within a sub-Saharan West African context, and thus can devise local adaptive responses and systems to flooding. The implication here is that such 'informal' locally implemented and owned systems can integrate with and supplement more formal government initiatives to collectively help Ghanaian flood-prone communities to meet global goals and targets on community resilience for disaster risk management and climate change adaptation. 
In sum, much of the research from this volume both demonstrates the need and advocates for alternative policies that intentionally use the formal-informal interface as a key entry point to bridging the research-policy gap and advancing urban integration. However, understanding and mapping those entry points requires local knowledge, particularly of the informal, and we would argue that the acquisition of that knowledge is aided by co-productive methods and tools like the ones discussed in this volume.

\section{Concluding thoughts}

While interest in and experience of knowledge co-production in Africa is on the rise, it still remains a novel approach to research, and requires further support as well as theorization. The importance of such work cannot be underestimated, as when successful, CityLabs and other modes of knowledge co-production create important alternative spaces for knowledge-sharing, participatory planning, and urban governance, thus representing an important vehicle by which to bring the wider community together in understanding the urban challenges that beset African cities. The use of co-productive methods also offers the opportunity to incorporate the voices and perspectives of communities who have routinely been forgotten in the quest for one-size-fits-all technical solutions to development challenges. That said, CityLabs are but one method that can be undertaken as part of knowledge co-production, and researchers must continue to deliberately and methodically engage with communities and a wide range of urban stakeholders to generate the kind of knowledge that is required to disrupt the power imbalances that continue to be the hallmark of citizen and state relationships in African cities.

\section{Notes}

1 For a more detailed overview of knowledge co-production, see Chapter 1 of this volume.

2 Notably, out of the six different countries covered in this book, only two (Ghana and South Africa) were considered to be 'free' as measured by the degree of civil liberties and political rights by the US-based NGO Freedom House at the time of completion of the work in 2019. Zambia and Kenya are considered as 'partly free', while Egypt and Angola are considered 'not free'. See: https://freedomhouse.org/. By contrast, Chapter 5 on Cairo and Johannesburg, shows that different degrees of freedom can still result in similar outcomes for the urban poor, as in both cities 'mechanisms and modes of participation and engagement with the state are largely dysfunctional'.

\section{References}

Bulkeley, H. (2006). Urban sustainability: learning from best practice? Environment and Planning A, 38, pp. 1029-1044. DOI: https://doi.org/10.1068/a37300

Duminy, J., Odendaal, N. \& Watson, V. (2014). The education and research imperatives of urban planning professionals in Africa. In: Parnell, S. \& Pieterse, E. (Eds.), Africa's urban revolution. Cape Town: UCT Press, pp. 184-199.

Mitlin, D. \& Bartlett, S. (2018). Editorial: co-production - key ideas. Environment \& Urbanization, 30(2), pp. 355-366. DOI: https://doi.org/10.1177/0956247818791931 
Patel, Z., Greyling, S., Parnell, S. \& Pirie, G. (2015). Co-producing urban knowledge: experimenting with alternatives to 'best practice'. International Development Planning Review, 37(2), pp. 187-203. DOI: https://doi.org/10.3828/idpr.2015.15

Swilling, M. (2014). Rethinking the science - policy interface in South Africa: experiments in knowledge co-production. South African Journal of Science, 110(5-6), pp. 1-7, Article \#2013-0265. DOI: 10.1590/sajs.2014/20130265

West, S.E., Büker, P., Ashmore, M., Njoroge, G., Welden, N., Muhoza, C., Osano, P., Makau, J., Njoroge, P. \& Apondo, W. (2020). Particulate matter pollution in an informal settlement in Nairobi: using citizen science to make the invisible visible. Applied Geography, 114. DOI: https://doi.org/10.1016/j.apgeog.2019.102133 